\title{
Doses de extrato pirolenhoso no cultivo de orquídea ${ }^{1}$
}

\author{
Jenniffer Aparecida Schnitzer ${ }^{* 2}$, Mei Ju Su${ }^{3}$, Mauricio Ursi Ventura, Ricardo Tadeu de Faria ${ }^{5}$ \\ http://dx.doi.org/10.1590/0034-737X201562010013
}

\begin{abstract}
RESUMO
O extrato pirolenhoso vem sendo utilizado para diversos fins na agricultura, como a melhoria do desenvolvimento vegetativo, a fertilização orgânica, o condicionamento do do solo e a indução de enraizamento. Este trabalho teve como objetivo avaliar o efeito de diferentes doses de extrato pirolenhoso no cultivo da orquídea Cattleya loddigesii Lindl. Utilizaram-se plantas propagadas in vitro, as quais foram cultivadas em vasos com substrato composto de fibra de coco, casca de pinus e casca de arroz carbonizada $(1: 1: 1 \mathrm{v} / \mathrm{v} / \mathrm{v})$. As regas foram realizadas manualmente duas vezes por semana, no outono e no inverno, e três vezes por semana, na primavera e no verão. Os tratamentos foram: 0,0 (controle), $0,1,0,2,0,3,0,4,0,5$ e $0,6 \%$, valores que correspondem a $0,1,2,3,4,5$ e $6 \mathrm{~mL} \mathrm{~L}^{-1}$ de extrato pirolenhoso, respectivamente, aplicados utilizando-se o produto diluído em água, no volume de $50 \mathrm{~mL}$ por vaso a cada 30 dias. Após 12 meses do início do experimento, foram avaliados altura da parte aérea, número de brotos, número de folhas, número de pseudobulbos, comprimento da maior raiz, número de raízes, comprimento da maior folha, massa fresca total, massa seca da parte aérea e $\mathrm{pH}$ do substrato. A análise química foliar foi realizada para os elementos cálcio, magnésio, fósforo, potássio e nitrogênio. Observou-se que a aplicação do extrato pirolenhoso foi eficaz no cultivo da espécie Cattleya loddigesii Lindl., sendo recomendada a dose de 0,6\%.
\end{abstract}

Palavras-chave: Ácido pirolenhoso, Cattleya, líquido pirolenhoso, Orchidaceae, vinagre de madeira.

\section{ABSTRACT}

\section{Pyroligneous extract doses in orchids culture}

Pyroligneous extract has been used in the cultivation of plants for various purposes in agriculture such as improvement of plant development, organic fertilization, soil conditioner and root induction. The objective of this study was to evaluate the effect of the use of different doses of pyroligneous extract in the cultivation of the orchid Cattleya loddigesii Lindl. In vitro propagated plants were used, grown in pots containing a substrate composed of coconut fiber, pine bark and carbonized rice husk $(1: 1: 1 \mathrm{v} / \mathrm{v} / \mathrm{v})$. Irrigation was carried out manually, twice a week in the fall and winter and three times a week in spring and summer. The treatments were as follows:: 0 (control); 0.1; $0.2 ; 0.3 ; 0.4 ; 0.5$ and $0.6 \%$, which corresponded to $0,1,2,3,4,5$ and $6 \mathrm{~mL} \cdot \mathrm{L}^{-1}$ of pyroligneous extract, respectively, which were applied using the product diluted in water at the volume of $50 \mathrm{~mL}$ per pot every 30 days. After 12 months from the beginning of the experiment, the following were evaluated: height of aerial part, number of buds, number of leaves, number of pseudobulbs, length of the longest root, number of roots,largest leaf length, total fresh mass, aerial part dry mass part, and substrate $\mathrm{pH}$. The leaf chemical analysis was performed for the following elements: calcium, magnesium, phosphorus, potassium and nitrogen. It was observed that the application of pyroligneous extract was effective in the cultivation of Cattleya loddigesii Lindl, being recommended the dose of $0.6 \%$.

Key words: Acid pyroligneous, Cattleya, liquid pyroligneous, Orchidaceae, vinager wood.

\footnotetext{
Submetido em 27/04/2011 e aprovado em 23/04/2014.

' Dissertação da primeira autora, apresentada à Universidade Estadual de Londrina para obtenção do título de Mestre.

${ }^{2}$ Universidade Estadual de Londrina, Londrina, Paraná, Brasil. je_uel@yahoo.com.br (Bolsista CNPq).

${ }^{3}$ Universidade Estadual de Londrina, Londrina, Paraná, Brasil. meijusu @ hotmail.com (Bolsista CNPq).

+Universidade Estadual de Londrina, Departamento de Agronomia, Londrina, Paraná, Brasil. mventura@uel.br

${ }^{5}$ Universidade Estadual de Londrina, Departamento de Agronomia, Londrina, Paraná, Brasil. faria@uel.br

Autor para correspondência: je_uel@yahoo.com.br
} 


\section{INTRODUÇÃO}

A família Orchidaceae está entre as plantas ornamentais mais apreciadas, por seu grande valor comercial, contando com aproximadamente 35.000 espécies e vários híbridos, sendo a maior e a mais evoluída família do reino vegetal (Suttleworth et al., 1970). O gênero Cattleya compreende cerca de setenta espécies e inúmeras variedades e híbridos, estando presente em todo o território brasileiro (Kämpf, 2000). A espécie Cattleya loddigesii Lindley encontra-se distribuída por diversas regiões tropicais das Américas do Sul e Central. Apresenta de três a seis flores, com 8 a $11 \mathrm{~cm}$ de envergadura e com espata na base. São plantas epífitas originárias de regiões com altitude elevada, ocorrendo em matas com elevado grau de umidade (Miller \& Warren, 1996).

O Brasil produz seis milhões de toneladas anuais de carvão vegetal, sua utilização renova-se tecnologicamente, apoiada na ampla disponibilidade de mão de obra e de terras apropriadas à exploração. O impacto ambiental da produção do carvão vegetal na poluição atmosférica é bastante alto em certos locais, principalmente nos vales e em certas épocas do ano, como no inverno, ainda que se obedeça às condições que regulamentam a localização dos fornos, o número de fornos por produtor e os mecanismos de amenização desse problema, como, por exemplo, a implantação de cortina vegetal (Encarnação, 2001).

Em 2001, o Estado do Rio Grande do Sul iniciou a implantação de sistemas de coleta de fumaça e produção de extrato pirolenhoso em fornos, com objetivo de reduzir a quantidade de fumaça lançada na atmosfera e obter um produto útil para os agricultores no controle e prevenção, principalmente de insetos, em várias culturas, além de outros benefícios do produto. Os resultados foram positivos, diminuindo aproximadamente $50 \%$ da quantidade de fumaça lançada na atmosfera (avaliação visual) e proporcionando a produção de 24 litros de extrato pirolenhoso bruto, em um forno de $6,0 \mathrm{~m}^{3} \mathrm{de}$ capacidade de madeira (Encarnação, 2001).

O extrato pirolenhoso (EP) é obtido, no processo de carbonização da madeira ou do bambu para a produção do carvão, a partir de um líquido formado pela condensação da fumaça durante a queima, sendo, também, conhecido como ácido pirolenhoso ou vinagre de madeira. O EP é composto, em sua maior parte, por água e mais de 200 compostos orgânicos, dentre os quais ácido acético, álcoois, cetonas, fenóis e alguns derivados de lignina. Esse produto pode ser obtido de diferentes espécies vegetais, como o bambu, o eucalipto e o pinus (Guirra, 2003; Alves, 2006; Campos 2007).

Segundo Miyasaka et al. (1999; 2001), o extrato pirolenhoso é conhecido e utilizado como condiciona- dor do solo (melhora as qualidades físicas, químicas e especialmente biológicas, proporcionando aumento de micro-organismos benéficos, facilitando, assim, a assimilação de nutrientes do solo pela planta), bioestimulante vegetal, indutor de enraizamento, repelente de insetos, contribuindo com a diminuição do uso de agroquímicos na agricultura convencional.

O extrato pirolenhoso pode ser utilizado para diversos fins na agricultura, como fertilizante orgânico em arroz (Ichikawa \& Ota, 1982; Shirakawa et al. 1993; Tsuzuki et al., 2000), melão (Tsuzuki et al., 1993; Du et al., 1997), cana-de-açúcar (Uddin et al., 1995), sorgo (Esechie et al., 1998) e batata-doce (Shibayama et al., 1998). No Brasil, alguns trabalhos foram desenvolvidos com o uso desse insumo em agricultura, como por exemplo, nas culturas do limoeiro 'cravo' (Zanetti et al., 2004), do eucalipto (Souza-Silva et al., 2006), da alface e do quiabo (Mascarenhas et al., 2006a, 2006b), de mudas de pinus (Porto et al., 2007), de alface (Roel et al., 2007), de couve-de-folha (Shingo \& Ventura, 2009), de orquídeas Cattleya intermedia e Miltonia clowesii, em que ocorreu um incremento do desenvolvimento vegetativo e radicular das espécies, ao utilizarem extrato pirolenhoso, acrescido ao substrato (Schnitzer et al., 2010) e de mudas de areca-bambu (Wanderley et al., 2012).

A sua utilização tem como finalidade proporcionar um aumento no desenvolvimento vegetativo no cultivo de plantas. Sendo assim, este trabalho foi desenvolvido com o objetivo de avaliar o efeito de diferentes doses de extrato pirolenhoso no cultivo da orquídea Cattleya loddigesii Lindl.

\section{MATERIAL E MÉTODOS}

O experimento foi conduzido no orquidário do Departamento de Agronomia do Centro de Ciências Agrárias (CCA) da Universidade Estadual de Londrina (UEL), Londrina -PR, $\left(23^{\circ} 23^{\prime}\right.$ de latitude Sul e $51^{\circ} 11^{\prime}$ de longitude Oeste; altitude média de $566 \mathrm{~m}$ ), de maio de 2007 a maio de 2008 .

A espécie utilizada foi Cattleya loddigesii Lindl, tendo as mudas, provenientes da propagação in vitro, dois anos e altura média de $12 \pm 1,0 \mathrm{~cm}$. As plantas foram cultivadas em telado de polipropileno, com retenção de $50 \%$ de fluxo de radiação solar. O plantio foi realizado em vasos plásticos pretos, com 15,0 cm de altura e 12,5 $\mathrm{cm}$ de diâmetro, com uma camada de $3 \mathrm{~cm}$ de pedra brita, no fundo, para facilitar a drenagem da água de irrigação.

O substrato utilizado foi uma mistura de fibra de coco, casca de pinus e casca de arroz carbonizada (Assis et al., 2008; Lone et al., 2008), em proporções iguais $(1: 1: 1 \mathrm{v} / \mathrm{v} / \mathrm{v})$. As regas foram realizadas manualmente, duas vezes por semana, no inverno, e três vezes por semana, no verão. 
Os tratamentos foram realizados por diluição do extrato pirolenhoso (Pirosfértilß) concentrado (100\%), em água destilada, e por aplicação direta no substrato de $50 \mathrm{~mL}$ por vaso, a cada 30 dias. $\mathrm{O}$ extrato pirolenhoso foi proveniente da Carvoaria Coroados (Londrina, PR), utilizando-se madeira de reflorestamento de Eucalyptus grandis W. Hill ex Maiden. A análise química dos elementos que constituíam o extrato pirolenhoso é apresentada na Tabela 1.

As concentrações de EP avaliadas foram: 0,0 (controle), $0,1,0,2,0,3,0,4,0,5$ e $0,6 \%$, valores que correspondem a $0,1,2,3,4,5$ e $6 \mathrm{~mL} \mathrm{~L}^{-1}$ de extrato pirolenhoso, respectivamente.

Um ano após a instalação do experimento, foram avaliadas as variáveis altura da parte aérea (APA), número de brotos (NB), número de folhas (NF), número de pseudobulbos (NP), comprimento da maior raiz (CMR), número de raízes (NR), comprimento da maior folha (CMF), massa fresca total (MFT) e massa seca da parte aérea (MSPA).

A análise química foliar dos elementos cálcio $(\mathrm{Ca})$, magnésio $(\mathrm{Mg})$, fósforo $(\mathrm{P})$, potássio $(\mathrm{K})$ e nitrogênio (N) e a determinação do potencial hidrogeniônico $(\mathrm{pH})$ do substrato foram realizadas conforme Silva (1999).

O delineamento experimental utilizado foi o inteiramente casualizado, com 12 repetições por tratamento. Para as variáveis números de raízes (NR) e número de folhas (NF), os dados foram transformados pela constante $\sqrt{\mathrm{X}+1}$, sendo que as médias apresentadas não são as transformadas. Os dados foram submetidos à análise de variância, ao teste de Tukey a 5\% e à análise de regressão.

\section{RESULTADOS E DISCUSSÃO}

De acordo com o teste de Tukey a 5\%, para a espécie Cattleya loddigesii, as médias do comprimento da maior raiz (CMR), do número de folhas (NF), do número de brotos (NB), da massa seca da parte aérea (MSPA), do potencial hidrogeniônico $(\mathrm{pH})$, (Tabela 2) e as médias referentes aos teores foliares de fósforo $(\mathrm{P})$, potássio $(\mathrm{K})$, cálcio $(\mathrm{Ca})$ e magnésio $(\mathrm{Mg})$ apresentaram resulta-

Tabela 1. Análise química do Extrato Pirolenhoso (Pirosfértilß), realizada no Laboratório Laborsolo - Londrina/PR, 2008

\begin{tabular}{lc}
\hline Parâmetros & Resultados $(\%)$ \\
\hline Nitrogênio Total & 3,500 \\
Potássio Total & 0,003 \\
Cobre Total & 0,090 \\
Zinco Total & 0,100 \\
Cálcio Total & 0,160 \\
Magnésio Total & 0,400 \\
Enxofre Total & 10,100 \\
Potencial hidrogeniônico $(\mathrm{pH})$ & 3,500 \\
\hline
\end{tabular}

dos similares entre os tratamentos, não sendo constatado efeito das doses de extrato pirolenhoso (Tabela 3).

Foram ajustadas equações de regressão, utilizandose, como variáveis dependentes, a percentagem de extrato pirolenhoso (EP) aplicado, sendo as características que apresentaram diferença entre os tratamentos a altura da parte aérea (APA), o número de raízes (NR), o número de pseudobulbos (NP), o comprimento da maior folha (CMF), a massa fresca total (MFT) e o teor foliar (N).

Para altura da parte aérea (APA), foi possível verificar que, conforme aumentavam as doses de extrato pirolenhoso, ocorria acréscimo da altura da parte aérea das plantas. Esses resultados estão de acordo com os de Schnitzer et al. (2010), que constataram aumento da altura da parte aérea da orquídea Cattleya intermedia quando utilizaram como substrato casca de pinus, fibra de coco, casca de arroz carbonizada e o carvão banhado no extrato pirolenhoso puro. Dados semelhantes foram observados, em condições de campo, por Ichikawa \& Ota (1982), que constataram melhor desenvolvimento da parte aérea das mudas de arroz com a aplicação do extrato pirolenhoso no solo. Ainda, a adição de EP na adubação orgânica isolada de areca-bambu proporcionou maiores alturas de planta (Wanderley et al., 2012).

Verificou-se aumento do número de raízes (NR), de acordo com o aumento das doses de EP, sendo a melhor dose a de 0,6\% de EP (Figura 1). Resultados semelhantes foram observados em estudo com mudas de Pinus elliottii, nos quais todos os tratamentos com EP apresentaram maior desenvolvimento radicular e foliar quando comparados ao controle (Porto et al. , 2007). Ichikawa \& Ota (1982), constataram que a aplicação de extrato pirolenhoso ao substrato proporcionou melhor enraizamento das plantas de arroz. Du et al. (1998), ao utilizarem a mistura de carvão vegetal com extrato pirolenhoso na cultura de batata doce, obtiveram aumento do crescimento das raízes. Uddin et al. (1995), utilizando a mistura de carvão vegetal e extrato pirolenhoso, constataram aumento do desenvolvimento das raízes na cultura de cana-de-açúcar. Schnitzer et al. (2010), utilizando como substrato casca de pinus, fibra de coco, casca de arroz carbonizada e o carvão banhado no extrato pirolenhoso puro, verificaram maior número de raízes em Cattleya intermedia.

$\mathrm{O}$ extrato pirolenhoso promoveu aumento do número de pseudobulbos (NP) diretamente proporcional às doses aplicadas, conforme se aumentavam as doses de EP ocorriam acréscimos do número de pseudobulbos, sendo a melhor a dose de 0,6\% de EP (Figura 2). Esse resultado indica que o produto faz aumentar o número de pseudobulbos e, consequentemente, o número de flores. De acordo com Assis (2003), o pseudobulbo exer- 
ce um importante papel na demanda energética da planta, uma vez que armazena água e carboidratos. O aumento do número de pseudobulbos garante a sustentação das folhas e flores e causa efeitos benéficos sobre o desenvolvimento vegetativo das plantas.

Analisando-se o comprimento da maior folha (CMF) foram observadas maiores médias quando se aplicou a dose estimada de $0,51 \%$ de EP.

Para a massa fresca total (MFT), observou-se que a aplicação da dose estimada de $0,57 \%$ de EP promoveu aumento da massa fresca total das plantas (Figura 3). Resultados semelhantes foram observados por Schnitzer et al. (2010) que verificaram aumento significativo da massa fresca total das espécies Cattleya intermedia e Miltonia clowesii, quando utilizaram casca de pinus, fibra de coco, casca de arroz carbonizada e carvão vegetal imerso no extrato pirolenhoso. De acordo com Du et al. (1998), a mistura de adubo orgânico com extrato pirolenhoso no cultivo de batata-doce resultou no aumento de tubérculos.

Tabela 2. Médias do comprimento da maior raiz (CMR), número de folhas (NF), número de brotos (NB), massa seca da parte aérea (MSPA) e potencial hidrogeniônico $(\mathrm{pH})$ de Cattleya loddigesii, submetidos a diferentes doses de extrato pirolenhoso, doze meses após a instalação do experimento. Londrina-PR, Brasil, 2011

\begin{tabular}{lccccc}
\hline Doses EP $(\boldsymbol{\%})$ & CMR $(\mathbf{c m})$ & NF & NB & MSPA $(\mathbf{m g})$ & pH \\
\hline 0,0 & $22,63 \mathrm{a}$ & $4,09 \mathrm{a}$ & $0,17 \mathrm{a}$ & $11,11 \mathrm{a}$ & $4,17 \mathrm{a}$ \\
0,1 & $20,39 \mathrm{a}$ & $3,95 \mathrm{a}$ & $0,33 \mathrm{a}$ & $7,83 \mathrm{a}$ & $4,80 \mathrm{a}$ \\
0,2 & $23,49 \mathrm{a}$ & $4,16 \mathrm{a}$ & $0,18 \mathrm{a}$ & $8,67 \mathrm{a}$ & $4,50 \mathrm{a}$ \\
0,3 & $29,03 \mathrm{a}$ & $4,13 \mathrm{a}$ & $0,50 \mathrm{a}$ & $7,03 \mathrm{a}$ & $4,26 \mathrm{a}$ \\
0,4 & $26,93 \mathrm{a}$ & $4,09 \mathrm{a}$ & $0,36 \mathrm{a}$ & $7,79 \mathrm{a}$ & $5,11 \mathrm{a}$ \\
0,5 & $25,56 \mathrm{a}$ & $4,73 \mathrm{a}$ & $0,36 \mathrm{a}$ & $6,47 \mathrm{a}$ & $4,74 \mathrm{a}$ \\
0,6 & $22,50 \mathrm{a}$ & $4,32 \mathrm{a}$ & $0,42 \mathrm{a}$ & $6,80 \mathrm{a}$ & $5,20 \mathrm{a}$ \\
\hline CV \% & 30,88 & 15,84 & 31,23 & 28,47 & 14,30 \\
\hline
\end{tabular}

Médias seguidas de mesma letra na coluna não diferem entre si pelo teste de Tukey a 5\% de significância.

* As médias apresentadas não são as transformadas.

Tabela 3. Médias referentes aos teores foliares de fósforo $(\mathrm{P})$, potássio $(\mathrm{K})$, cálcio $(\mathrm{Ca})$ e magnésio $(\mathrm{Mg})$ Cattleya loddigesii, submetidos a diferentes doses de extrato pirolenhoso, 12 meses após a instalação do experimento. Londrina-PR, Brasil, 2011

\begin{tabular}{lcccc}
\hline Doses EP $(\%)$ & $\mathbf{P}\left(\mathbf{g ~ K g} \mathbf{~}^{-1}\right)$ & $\mathbf{K}\left(\mathbf{g ~ K} \mathbf{~}^{-1}\right)$ & $\mathbf{C a}\left(\mathbf{g ~ K g}^{-1}\right)$ & $\mathbf{M g}\left(\mathbf{g ~ K \mathbf { ~ g } ^ { - 1 } )}\right.$ \\
\hline 0,0 & $0,04 \mathrm{a}$ & $128,76 \mathrm{a}$ & $10,82 \mathrm{a}$ & $0,63 \mathrm{a}$ \\
0,1 & $0,03 \mathrm{a}$ & $142,66 \mathrm{a}$ & $10,87 \mathrm{a}$ & $1,09 \mathrm{a}$ \\
0,2 & $0,04 \mathrm{a}$ & $116,84 \mathrm{a}$ & $9,34 \mathrm{a}$ & $0,97 \mathrm{a}$ \\
0,3 & $0,04 \mathrm{a}$ & $132,73 \mathrm{a}$ & $11,88 \mathrm{a}$ & $0,50 \mathrm{a}$ \\
0,4 & $0,03 \mathrm{a}$ & $107,58 \mathrm{a}$ & $14,29 \mathrm{a}$ & $0,63 \mathrm{a}$ \\
0,5 & $0,04 \mathrm{a}$ & $108,24 \mathrm{a}$ & $12,11 \mathrm{a}$ & $1,34 \mathrm{a}$ \\
0,6 & $0,04 \mathrm{a}$ & $127,44 \mathrm{a}$ & $8,27 \mathrm{a}$ & $0,71 \mathrm{a}$ \\
\hline CV \% & 24,39 & 11,80 & 23,24 & 30,20 \\
\hline
\end{tabular}

Médias seguidas de mesma letra na coluna não diferem entre si pelo teste de Tukey a 5\% de significância.

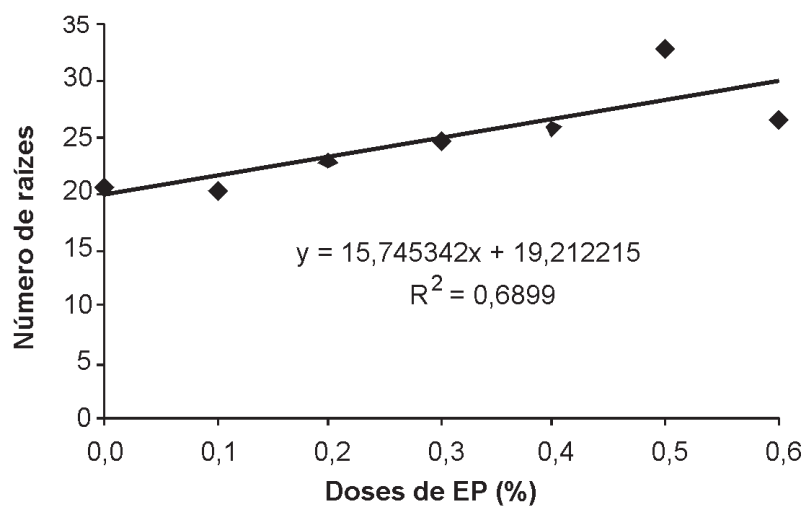

Figura 1. Número de raízes da orquídea Cattleya loddigesii Lindl., com diferentes doses de extrato pirolenhoso.

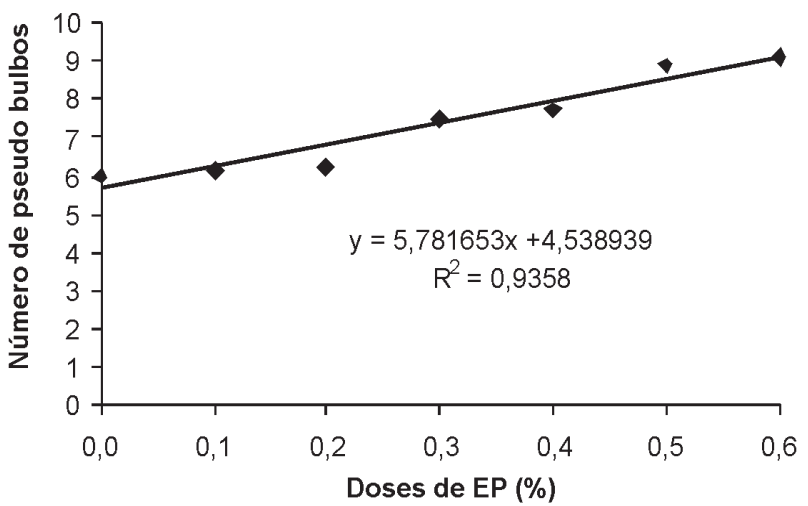

Figura 2. Número de pseudobulbos da orquídea Cattleya loddigesii Lindl., com diferentes doses de extrato pirolenhoso. 


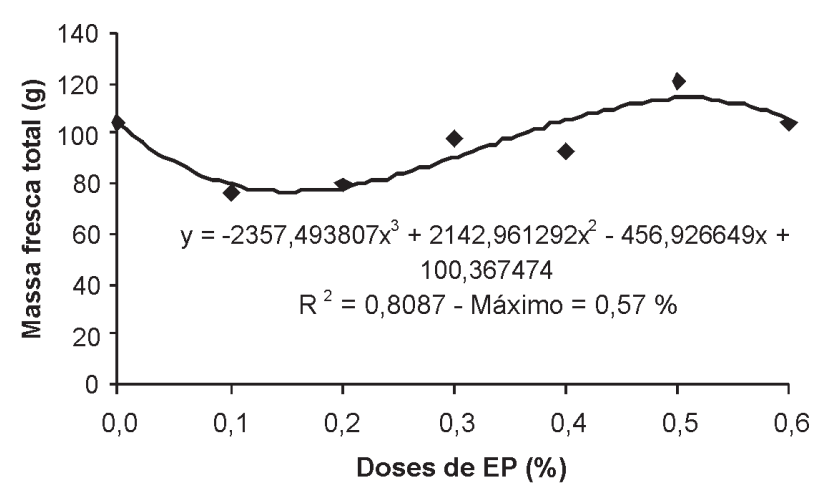

Figura 3. Massa fresca total da orquídea Cattleya loddigesii Lindl., com diferentes doses de extrato pirolenhoso.

Por meio das análises químicas, foi possível verificar teores de nitrogênio $(\mathrm{N})$ foliar mais elevados com a aplicação da dose estimada de 0,30\% de EP (Figura 4). $\mathrm{O}$ aumento dos teores de $\mathrm{N}$ foliar garante um bom desenvolvimento das plantas, sendo este elemento, constituinte de muitos componentes da célula vegetal, fundamental para o crescimento (Taiz \& Zeiger, 2004).

Os resultados obtidos com a Cattleya loddigesii corroboram os de vários autores, em trabalhos que demonstraram efeito benéfico do extrato pirolenhoso para diversas culturas. Por exemplo, Kadota \& Niimi (2004), ao trabalharem com as espécies ornamentais Tagetes patula, Melampodium paludosum, Salvia splendens e Zinnia linearis, verificaram que, em tratamento com carvão vegetal e esterco, ocorreram melhor crescimento e maior qualidade das mudas de Zinnia linearis tratadas com carvão vegetal e extrato pirolenhoso a 0 - 10 e $30 \% \mathrm{v} / \mathrm{v}$, ocorrendo diminuição do número de dias para florescimento de Zinnia linearis e Melampodium paludosu.

Os resultados obtidos mostram que o extrato pirolenhoso é benéfico para o desenvolvimento vegetativo da espécie estudada, sendo uma alternativa na nutrição de plantas, como bioestimulante vegetal e indutor de enraizamento.

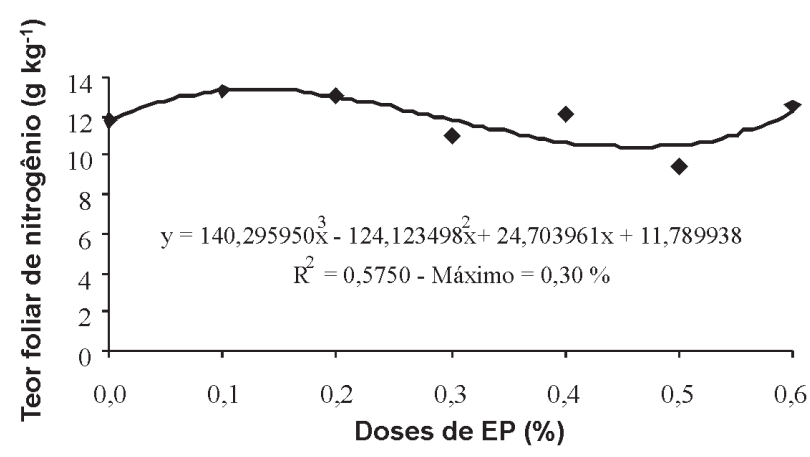

Figura 4. Teor de nitrogênio foliar da orquídea Cattleya loddigesii Lindl., com diferentes doses de extrato pirolenhoso.

\section{CONCLUSÕES}

O extrato pirolenhoso é eficaz para o cultivo de Cattleya loddigesii Lindl.

A dose de extrato pirolenhoso (EP) recomendada para a espécie Cattleya loddigesii Lindl. é de 0,6\% $\left(6 \mathrm{~mL} \mathrm{~L}^{-1}\right)$.

\section{AGRADECIMENTOS}

Os autores agradecem ao Conselho Nacional de Desenvolvimento Científico e Tecnológico (CNPq), pela concessão de bolsa.

\section{REFERÊNCIAS}

Alves M (2006) Impactos da utilização de fino de carvão e extrato pirolenhoso na agricultura. Dissertação de Mestrado. Universidade Estadual Paulista "Julio de Mesquita Filho", Jaboticabal. 52p.

Assis AM, Colombo LA, Faria RT \& Fonseca ICB (2003) Longevidade pós-colheita de pseudobulbos com flores de Dendrobium nobile (Orchidaceae). Revista Brasileira de Horticultura Ornamental, 9:8587.

Assis AM, Faria RT, Unemoto LK \& Colombo LA (2008) Cultivo de Oncidium baueri Lindley (Orchidaceae) em substratos a base de coco. Ciência e Agrotecnologia, 32:981-985.

Campos AD (2007) Técnicas para produção de extrato pirolenhoso para uso agrícola. Pelotas, Embrapa CPACT. 8p. (Circular Técnica, 65).

Du HG, Mori E, Terao H \& Tsuzuki E (1998) Effect of the mixture of charcoal with pyroligneous acid on shoot and root growth of sweet potato [Ipomoea batatas]. Japanese Journal of Crop Science, 67:149-152.

Du HG, Ogawa M, Ando S, Tsuzuki E \& Murayama S (1997) Effect of mixture of charcoal with pyroligneous acid on sucrose content in netted melon (Cucumis melo L. var. reticulates Naud.) fruit. Japanese Journal of Crop Science, 66:369-373.

Encarnação F (2001) Redução do impacto ambiental na produção de carvão vegetal e obtenção do ácido pirolenhoso como alternativa para proteção de plantas. Agroecologia e Desenvolvimento Rural Sustentável, 2:20-23.

Esechie HA, Dhaliwal GS, Arora L, Randhawa NS \& Dhawan AK (1998) Assessment of pyroligneus liquid as a potential organic fertilizer. In: $1^{\circ}$ Ecological Agriculture and Sustainable Development, Chandigarh. Proceedings, Center for Research in Rural and Industrial Development. p.591-595.

Guirra L (2003) Agricultores conhecem benefícios do carvão e do extrato pirolenhoso. Disponível em: <www.guirra.com.br/guirranet/ pirolenhosso.htm> Acessado em: 16 de junho de 2007 .

Ichikawa T \& Ota Y (1982) Effect of pyroligneous acid on the growth of rice seedlings. Japanese Journal of Crop Science, 51:14-17.

Kadota M \& Niimi Y (2004) Effects of charcoal with pyroligneous acid and barnyard manure on bedding plants. Scientia Horticulturae, 101:327-332.

Kämpf AN (2000) Produção comercial de plantas ornamentais. Guaíba, Agropecuária. 254p.

Lone AB, Barbosa CM, Takahashi LSA \& Faria RT (2008) Aclimatização de Cattleya (Orchidaceae), em substratos alternativos ao xaxim e ao esfagno. Acta Scientiarum Agronomy, 30:465-469.

Mascarenhas MHT, Lara JFR, Purcino HMA, Simões JC, Moreira DC \& Facion CE (2006a) Efeito da utilização do extrato pirolenhoso na produtividade do alface. Revista Brasileira de Horticultura, 24:3122-3125. 
Mascarenhas MHT, Lara JFR, Purcino HMA, Simões JC, Moreira DC \& Facion CE (2006b) Efeito da utilização do extrato pirolenhoso na produtividade do quiabeiro. Revista Brasileira de Horticultura, 24:3126-3128.

Miller D \& Warren R (1996) Orquídeas do Alto da Serra. Rio de Janeiro, Salamandra. 256p.

Miyasaka S, Ohkawara T \& Utsumi B (1999) Ácido Pirolenhoso: uso e fabricação. Botucatu. (Boletim AgroEcológico, 14).

Miyasaka S, Ohkawara T, Nagai K, Yazaki H \& Sakita MN (2001) Técnicas de produção e uso do fino de carvão e licor pirolenhoso. In: Encontro de Processos de Proteção de Plantas: Controle Ecológico de Pragas e Doenças, Botucatu. Resumo, FCA. p.161-179.

Porto PR, Sakita AEN \& Nakaoka SM (2007) Efeito da aplicação do extrato pirolenhoso na germinação e no desenvolvimento de mudas de Pinus elliottii var. elliottii. IF-Série Registros, 31:15-19.

Roel AR, Leonel LAK, Favaro SP, Zatarim M \& Soares MV (2007) Avaliação de fertilizantes orgânicos na produção de alface em Campo Grande, MS. Scientia Agraria, 8:325-329.

Silva FC (1999) Manual de análises químicas de solos, plantas e fertilizantes. Brasília, Embrapa Solos. 370p.

Souza-Silva AS, Zanetti R, Carvalho GA \& Mendonça LA (2006) Qualidade de mudas de eucalipto tratadas com extrato pirolenhoso. Cerne, 12:19-26.

Suttleworth FS, Zimmerman HS, Dillon GW \& Smith EW (1970) Orchids São Francisco, Western Publishing Company. 161p.

Schnitzer JA, Faria RT, Ventura MU \& Sorace M (2010) Substratos e extrato pirolenhoso no cultivo de orquídeas brasileiras (Cattleya intermedia (John Lindley) e Miltonia clowesii (John Lindley) (Orchidaceae). Acta Scientiarum Agronomy, 32:139-143.
Shibayama H, Mashima K, Mitsutomi M \& Arima S (1998) Effects of application of pyroligneus acid solution produced in Karatsu city and growth and free sugar contents of storage roots of sweet potatoes. Marine and Higland Bioscience Center Report, 7:15-23.

Shingo GY \& Ventura MU (2009) Produção de couve brassica oleracea L. var. acephala com adubação mineral e orgânica. Semina Ciências Agrárias, 30:589-594.

Shirakawa N, Fukazawa M \& Terada S (1993) Studies on the pyroligneous acid IV. Plant physiological activities of several main components in pyroligneous acid. Japanese Journal Crop Science, 62:168-189.

Taiz L \& Zeiger E (2004) Fisiologia vegetal. $3^{\mathrm{a}}$ ed. Porto Alegre, Artmed. $719 \mathrm{p}$

Tsuzuki E, Ando S, Terao H \& Uchida Y (1993) Effect of organic matters on growth and quality of crops: II Effect of charcoal with pyroligneous acid on quality of melon (Cucumis melo L.). Japanese Journal of Crop Science, 62:170-171.

Tsuzuki E, Morimtsu T \& Matsui T (2000) Effect of chemical compounds in pyroligneus acid on root growth in rice plants. Japanese Journal Crop Science, 66:15-16.

Uddin SMM, Murayama S, Ishimine Y, Tsuzuki E \& Harada J (1995) Effects of the mixture of charcoal with pyroligneous acid on dry matter production and root growth of summer planted sugarcane (Saccharum officinarum L.). Japanese Journal of Crop Science, 64:747-753.

Zanetti M, Cazetta JO, Mattos Júnior D \& Carvalho SA (2004) Influência do extrato pirolenhoso na calda de pulverização sobre o teor foliar de nutrientes em Limoeiro 'Cravo'. Revista Brasileira de Fruticultura, 26:529-533.

Wanderley CS, Faria RT \& Ventura MU (2012) Chemical fertilization, organic fertilization and pyroligneous extract in the development of seedlings of areca bamboo palm (Dypsis lutescens). Acta Scientiarum Agronomy, 34:163-167. 